\title{
Highly symmetric aperiodic structures -INVITED
}

\author{
Uwe Grimm ${ }^{1, *}$ \\ ${ }^{1}$ School of Mathematics and Statistics, The Open University, Walton Hall, Milton Keynes MK7 6AA, United Kingdom
}

\begin{abstract}
The symmetries of periodic structures are severely constrained by the crystallographic restriction. In particular, in two and three spatial dimensions, only rotational axes of order 1,2, 3, 4 or 6 are possible. Aperiodic tilings can provide perfectly ordered structures with arbitrary symmetry properties. Random tilings can retain part of the aperiodic order as well the rotational symmetry. They offer a more flexible approach to obtain homogeneous structures with high rotational symmetry, and might be of particular interest for applications. Some key examples and their diffraction are discussed.
\end{abstract}

\section{Introduction}

Motivated by the discovery of quasicrystals in intermetallic alloys by Dan Shechtman in 1982 [1], the investigation of aperiodic tilings and points sets has become an increasingly active area of mathematical research. One of the key fundamental questions from crystallography is how to properly define the concept of order, which is challenging, as can be seen from the ongoing discussion concerning the definition of a crystal; see [2] for background. While quasicrystals are now considered aperiodic crystals displaying aperiodic order, there is no clear demarcation line; in particular, there are many potential manifestations of partial order that may or may not be included.

The main tool to describe order in structures is linked to the experimental tool that is used to determine the structure of crystals, which is diffraction. Mathematical diffraction is a measure that is obtained by Fourier transform of the appropriately normalised pair correlation measure, and corresponds to the experimental diffraction in the kinematic far-field (Fraunhofer) limit; see [3] for details. Pure point diffraction is seen as the hallmark of an ordered structure, while continuous components, and in particular absolutely continuous components, are considered a sign of disorder. Traditionally, singular continuous components did not play a major role, but they have become more prominent in the discussion of aperiodic structures, because they are prevalent in substitution (or inflation) based structures displaying some self-similarity properties. A related approach to quantify order, which has recently become popular, is the concept of hyperuniformity [4], which is related to a scaling property of the diffraction measure; see [5] and references therein.

Aperiodic tilings and point sets not only serve as models for the atomic structure of quasicrystals, but are becoming increasingly important as metamaterials. The possibility to realise higher symmetries than in the severely

*e-mail: uwe.grimm@open.ac.uk constrained setting of periodic structures makes them particularly interesting for applications where isotropic properties are desirable [6]. With the recent advances in additive manufacturing, this opens up potential applications for metamaterials at any length scales, for applications exploiting their optical, phononic or mechanical properties.

In this brief note, we shall discuss some paradigmatic planar structures, focussing on their symmetry and diffraction. For mathematical and crystallographic background on the diffraction of aperiodic structures we refer to [7-9] and references therein.

\section{Cut and project and random tilings}

Cut and project sets (or model sets in the mathematical terminology) are obtained by projecting part of a higherdimensional periodic lattice. While there is are crystallographic restrictions for any dimensions, the number of possible symmetries increases with dimension. In particular, four-dimensional lattices account for axes with fivefold, eightfold, tenfold or twelvefold symmetries, and sixdimensional lattices can accommodate icosahedral symmetry as observed in physical quasicrystals.

Due to the underlying higher-dimensional periodic structure, these systems are generally well understood, provided some conditions on the projected subset of the lattice are satisfied. In particular, all cut and project sets satisfying these conditions are pure point diffractive, and the diffraction is concentrated on the projection of the dual (or reciprocal) lattice. As an example, the AmmannBeenker tiling is shown in figure 1. It can be obtained as a projection from the four-dimensional hypercubic lattice $\mathbb{Z}^{4}$. The corresponding diffraction measure for the infinite tiling is a pure point measure with perfect eightfold symmetry which is supported on a dense set in the plane. Figure 1 shows the diffraction image obtained for the finite patch, which already closely resembles the diffraction of the infinite structure. 

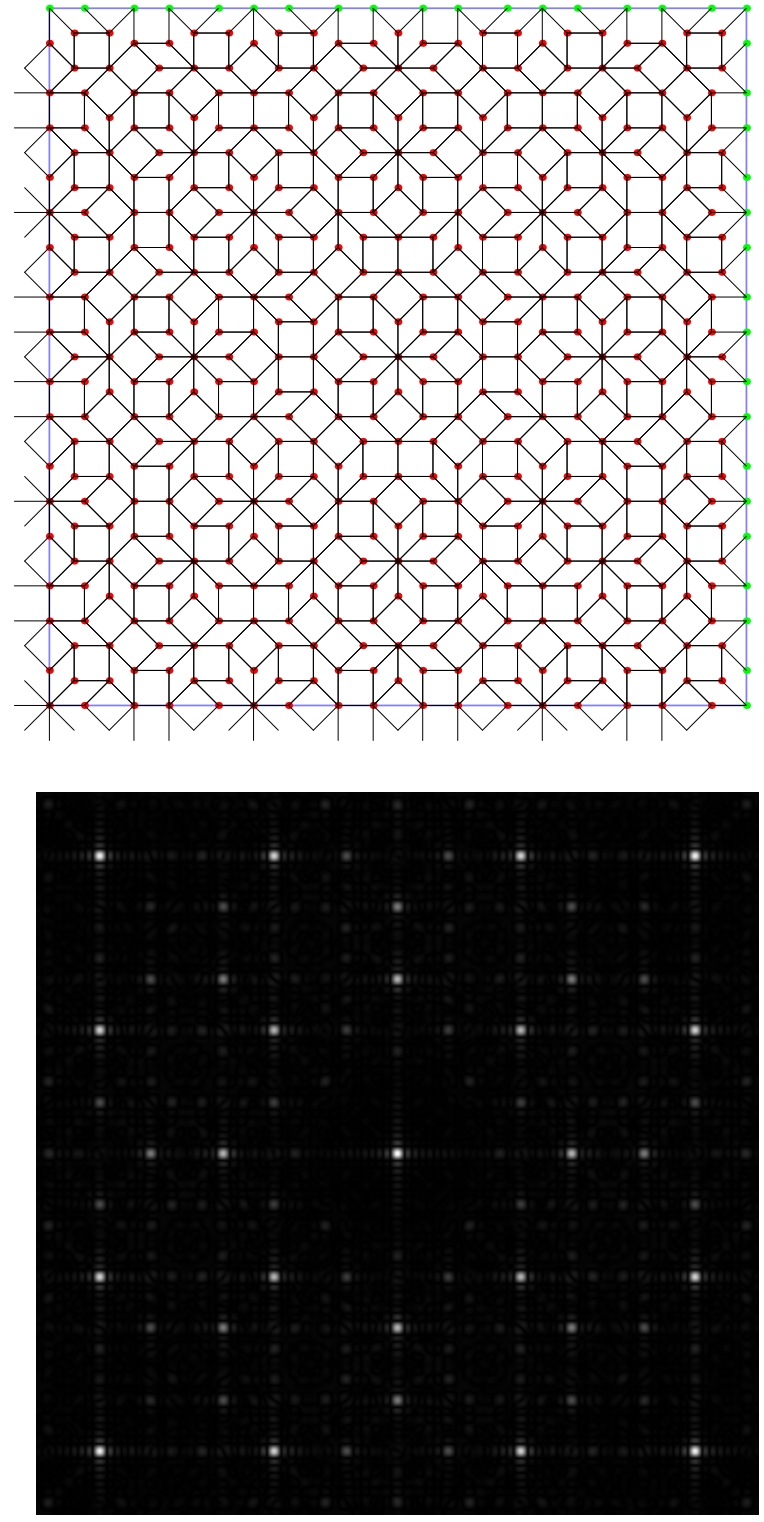

Figure 1. Finite patch of the Ammann-Beenker tiling (top) and its diffraction (bottom)

One way to introduce some disorder into the structure is by considering a random tiling, or more precisely the ensemble of all tilings of the plane with the same set of tiles. It turns out that the proportion of the two tiles in the eightfold tiling are optimal in the sense that they maximise the entropy of the tiling. Figure 2 shows an example of a randomised perfect tilings obtained by performing local flips (which are ergodic) of the tiles around vertices with three neighbours. The corresponding diffraction image (of this finite patch) is also shown in figure 2.

Since the random tiling preserves the eightfold symmetry (any finite patch is equally likely to occur in any orientation), the diffraction of the random tiling still shows perfect eightfold symmetry. Rigorous mathematical proofs for the nature of the diffraction of a random tiling are lacking, but heuristic arguments point to a singular continuous diffraction in the planar case. Note that the
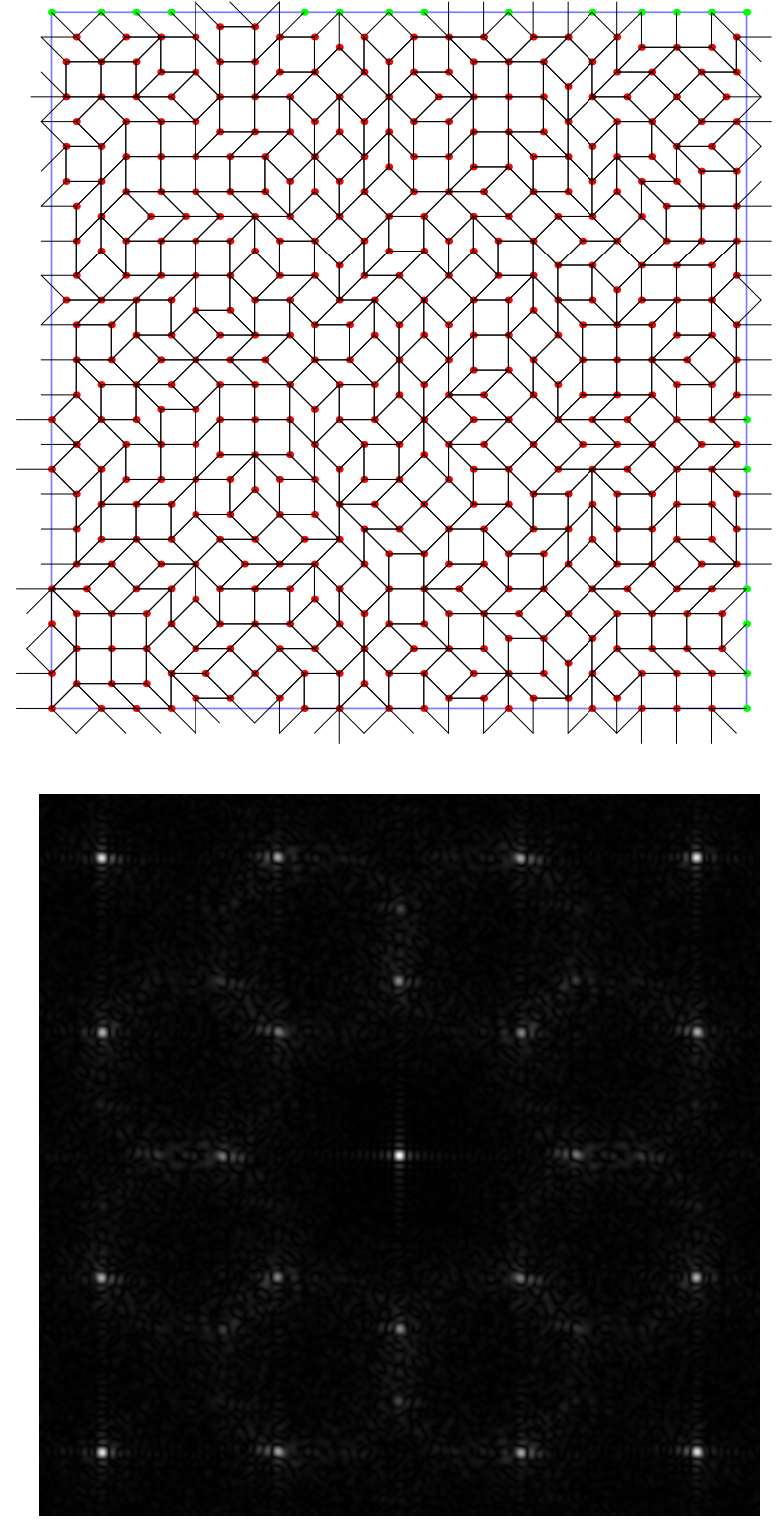

Figure 2. Finite patch of the Ammann-Beenker random tiling (top) and its diffraction (bottom)

random tiling can still be lifted into a subset of $\mathbb{Z}^{4}$, but this lift will, in general, no longer satisfy the conditions that guarantee pure point diffraction. Random tilings provide one way to obtain partially ordered structures with high rotational symmetries, and with a diffraction which is less strongly localised.

\section{Inflation tilings}

Inflation tilings are obtained by an iterative procedure consisting of a rescaling of the tiles followed by the dissection of the rescaled tiles into tiles of the original size. The resulting tilings possess self-similar properties. There are cut and project sets, such as the Ammann-Beenker tiling, that also possess an inflation symmetry, but in general cut and project sets do not possess an inflation symme- 
try, nor can general inflation tilings be lifted into a higherdimensional lattice.

Planar inflation tilings can also realise any rotational symmetry, and, maybe surprisingly, can even possess an infinite rotational symmetry, making them completely isotropic. The paradigm for the latter is the pinwheel tiling which is obtained from the inflation rule of figure 3 .

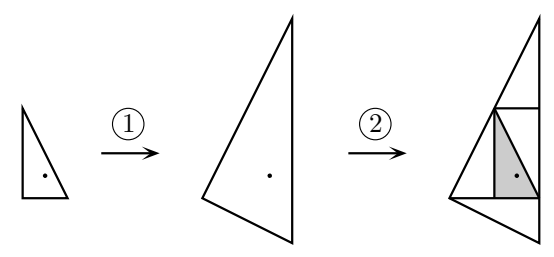

Figure 3. Inflation rule of the pinwheel tiling, consisting of a rescaling by a linear factor of $\sqrt{5}$ (step 1 ) followed by a dissection into five triangles of the original size (step 2). The rotation ensures that the shaded triangle has the same orientation as the original one, which ensures that iterating the rule can be used to define an infinite tiling; see [3] for details

The tile is a right triangle of side lengths 1,2 and $\sqrt{5}$. Each inflation step produces edges in a new direction in the plane. A patch of the pinwheel tiling obtained by inflating an initial patch consisting of two triangles joined along their hypotenuses is shown in figure 4 . Note that the two triangles are mapped onto each other by reflection, and that the reflected triangle is dissected by the reflected rule of that shown in figure 3, so that reflection symmetry is preserved.

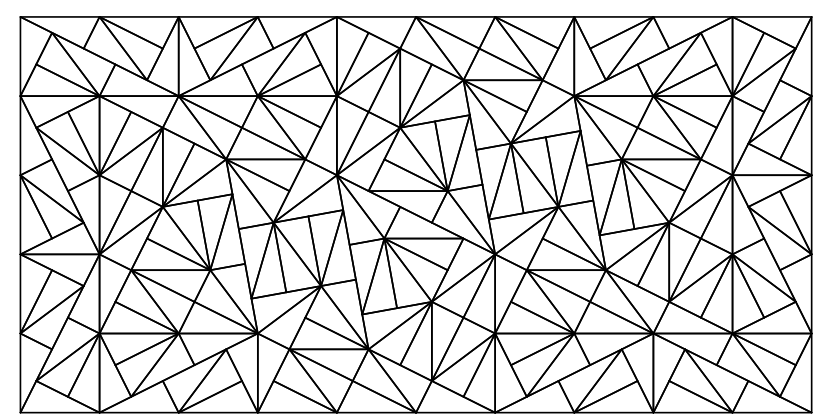

Figure 4. Patch of the pinwheel tiling obtained by three inflation steps from a couple of triangular tiles. It consists of $2 \times 5^{3}=250$ triangular tiles

The challenge with this tiling is that in each inflation step the number of tiles grows by a factor of five, while only one new direction is obtained. This means that any reasonably-sized finite patch will still be far from rotationally symmetric.

It has been proven that the diffraction measure for this tiling is fully rotationally symmetric, though it is still not clear whether it consists of sharp rings only (which amount to a singular continuous measure) or whether there is also an absolutely continuous component [10]. Due to the slow convergence to a rotationally symmetric structure, the investigation of finite patches does not provide sufficient evi- dence for or against the presence of absolutely continuous diffraction. However, recent results point towards weak hyperuniformity of the pinwheel tiling [11].

There are many other tilings with complete rotational symmetry, but the pinwheel tiling is arguable the simplest of these, using a single shape. In view of applications, which would necessarily use finite patches of such tilings, it is however less obvious whether these tilings really offer higher isotropy than tilings with finite rotational symmetry, such as the Ammann-Beenker tiling or a corresponding random tiling. For fivefold, eightfold, tenfold and twelvefold symmetry, the rotational symmetry can already be well realised in a rather small patch, as can be seen from the diffraction images in figures 1 and 2, while comparatively large patches of the pinwheel tiling may still be quite far from isotropic. The reason that these particular symmetries work well lies in the nature of the linear inflation factor for these cases, which is a quadratic PisotVijayaraghavan (PV) number; see [3] for details. At the same time, these are symmetries that can be realised in cut and project sets obtained from four-dimensional lattices, as mentioned above. They are also the planar symmetries that have been observed to occur in quasicrystalline materials $[12,13]$, so are also preferred by nature; so far, no material with other non-crystallographic symmetries have been found.

\section{Summary and Outlook}

While the class of cut and project tilings is generally well understood, this is much less so for tilings obtained by an inflation procedure, or indeed for any structures that involve randomness, such as random tilings. There exists a vast realm of unexplored structures which possess a degree of (partial) order, and a proper classification of ordered structures is nowhere in sight, leaving lots of opportunities for mathematical as well as application-oriented research. Random tilings with non-crystallographic symmetries and stochastic long-range order offer a large class of promising structures that has not yet received as much attention as it deserved.

Acknowledgements. This work was supported by Engineering and Physical Sciences Research Council (EPSRC) via grants EP/S010335/1 and EP/V047108/1.

\section{References}

[1] D. Shechtman, I. Blech, D. Gratias and J. W. Cahn, Metallic phase with long-range orientational order and no translational symmetry, Phys. Rev. Lett. 53, 19511953 (1984)

[2] U. Grimm, Aperiodic crystals and beyond, Acta Crystallogr. B 71, 258-274 (2015)

[3] M. Baake and U. Grimm, Aperiodic Order. Vol. 1: A Mathematical Invitation (Cambridge University Press, Cambridge, 2013)

[4] S. Torquato and F. H. Stillinger, Local density fluctuations, hyperuniformity, and order metrics, Phys. Rev. 
E 68, 041113 (2003) and Phys. Rev. E 68, 069901 (2003) (erratum)

[5] M. Baake and U. Grimm, Scaling of diffraction intensities near the origin: Some rigorous results, J. Stat. Mech.: Theory Exp. 2019, 054003 (2019)

[6] L. Dal Negro, Waves in Complex Media (Cambridge University Press, Cambridge, 2021)

[7] M. Baake and U. Grimm, Kinematic diffraction from a mathematical viewpoint, Z. Kristallogr. 226, 711-725 (2011)

[8] M. Baake and U. Grimm, Mathematical diffraction of aperiodic structures, Chem. Soc. Rev. 41, 6821-6843 (2012)

[9] M. Baake and U. Grimm, Inflation versus projection sets in aperiodic systems: The role of the window in averaging and diffraction, Acta Crystallogr. A 76,

$$
\text { 559-570 (2020) }
$$

[10] M. Baake, D. Frettlöh and U. Grimm, A radial analogue of Poisson's summation formula with applications to powder diffraction and pinwheel patterns, J. Geom. Phys. 57, 1331-1343 (2007)

[11] F. Sgrignuoli and L. Dal Negro, Hyperuniformity and wave localization in pinwheel scattering arrays, Phys. Rev. B 103, 224202 (2021)

[12] W. Steurer, Twenty years of structure research on quasicrystals. Part I. Pentagonal, octagonal, decagonal and dodecagonal quasicrystals, Z. Kristallogr. 219, 391-446 (2004)

[13] W. Steurer and S. Deloudi, Crystallography of Quasicrystals: Concepts, Methods and Structures (Springer, Heidelberg, 2009) 